\title{
Induction of Podocyte-Derived VEGF Ameliorates Podocyte Injury and Subsequent Abnormal Glomerular Development Caused by Puromycin Aminonucleoside
}

\author{
JI MA, TAIJI MATSUSAKA, HAI-CHUN YANG, JIANYONG ZHONG, NOBUAKI TAKAGI, AGNES B. FOGO, VALENTINA KON, \\ AND IEKUNI ICHIKAWA
}

\begin{abstract}
Departments of Pediatrics [J.M., J.Z., N.T., V.K., I.I.] and Pathology [H-C.Y., A.B.F.], Vanderbilt University Medical Center, Nashville, Tennessee 37232; Institute of Medical Science and Department of Internal Medicine [T.M.], Tokai University School of Medicine, Isehara, Kanagawa 259-1193, Japan
\end{abstract}

\begin{abstract}
Our previous studies using puromycin aminonucleoside (PAN) established that podocyte damage leads to glomerular growth arrest during development and glomerulosclerosis later in life. This study examined the potential benefit of maintaining podocyte-derived VEGF in podocyte defense and survival after PAN injury using conditional transgenic podocytes and mice, in which human VEGF-A (hVEGF) transgene expression is controlled by tetracycline responsive element (TRE) promoter and reverse tetracycline transactivator (rtTA) in podocytes. In vitro experiments used primary cultured podocytes harvested from mice carrying podocinrtTA and TRE-hVEGF transgenes, in which hVEGF can be induced selectively. Induction of VEGF in PAN-exposed podocytes resulted in preservation of intrinsic VEGF, $\alpha$-actinin-4 and synaptopodin, antiapoptotic marker Bcl-xL/Bax, as well as attenuation in apoptotic marker cleaved/total caspase-3. In vivo, compared with genotype controls, PAN-sensitive neonatal mice with physiologically relevant levels of podocyte-derived VEGF showed significantly larger glomeruli. Furthermore, PAN-induced up-regulation of desmin, downregulation of synaptopodin and nephrin, and disruption of glomerular morphology were significantly attenuated in VEGF-induced transgenic mice. Our data indicate that podocyte-derived VEGF provides self-preservation functions, which can rescue the cell after injury and preempt subsequent deterioration of the glomerulus in developing mice. (Pediatr Res 70: 83-89, 2011)
\end{abstract}

A nimal models and clinical studies indicate that podocyte damage is a key step in progression of glomerular injury to glomerular sclerosis (1-3). Although podocyte damage involves oxidative stress, mechanical overstretch, and deficiency of slit diaphragm proteins and/or toxins, our understanding of the intrinsic protective mechanisms for podocyte preservation and repair associated with injury remains largely unknown.

Vascular endothelial growth factor-A (VEGF-A) is an angiogenic and survival factor for endothelial cells $(4,5)$, primarily expressed in glomerular podocytes (6). Tight regulation of podocyte-derived VEGF is required for structural integrity of the glomerular endothelium $(7,8)$. VEGF down-

Received September 1, 2010; accepted January 25, 2011.

Correspondence: Ji Ma, M.D., Ph.D., Division of Pediatric Nephrology, Vanderbilt University Medical Center, MCN C4204, 1161 21st Avenue, Nashville, Tennessee 37232; e-mail: ji.ma@vanderbilt.edu

Supported by National Institute of Diabetes and Digestive and Kidney Diseases (DK037868 and DK044757) and American Heart Association (09BGIA2261364). regulation prevails in animal models $(9-11)$ and human diseases (12-14) where podocyte damage is thought to be intermediary in disease progression that culminates in glomerular injury and glomerulosclerosis (15-18). Supplementation of exogenous VEGF has been shown to attenuate glomerular lesions in remnant kidneys (9). Recently, we showed that damage in a portion of the podocyte population causes damage in the remaining podocytes, creating an autonomous vicious cycle that promotes progressive damage and loss of podocytes (19). We speculate that podocyte-derived VEGF functions as its own pivotal protective and survival factor after injury and have directly tested this hypothesis by enhancing podocyte VEGF that would otherwise be down-regulated upon exposure to insult. For this purpose, we studied puromycin aminonucleoside (PAN), a recognized podocyte toxin (20). Our previous study found that adult mice are resistant to PAN injury while PAN-injured podocytes cause underdeveloped glomeruli in neonatal mice (21). In this study, by using PAN-injured primary mouse podocytes and neonatal mice (Fig. 1), we demonstrated an autocrine podocyte survival effect of VEGF.

\section{METHODS}

Animals. Conditional transgenic mice are generated, in which human VEGF (hVEGF) transgene expression is controlled by tetracycline responsive element (TRE) promoter and reverse tetracycline transactivator (rtTA) in podocytes upon presence of tetracycline derivative, doxycycline (Dox). Mice double-transgenic for podocin-rtTA and TRE-hVEGF (Tg) $(22,23)$ were obtained from heterozygous mating. WT or podocin-rtTA single transgenic littermates served as genotype control $(\mathrm{Ctrl})$. Both lines were backcrossed to C57BL6 (Jackson Laboratory, Bar Harbor, ME) for more than 15 generations. Animal protocols were approved by Vanderbilt University Medical Center Institutional Animal Care and Use Committee in accordance with National Institutes of Health guidelines.

Isolation of glomeruli and podocyte culture. Glomeruli were isolated from 5- to 9-wk-old Tg and Ctrl mice using a modified methods of iron beads perfusion (Invitrogen, Carlsbad, CA), collagenase A dissociation (Roche Applied Science, Germany), and sieving (BD Falcon, Bedford, MA) (24,25),

Abbreviations: Ctrl, genotype control; Dox, doxycycline; Flt1, fms-like tyrosine kinase 1; hVEGF, human VEGF-A; mVEGF, mouse VEGF-A; PAN, puromycin aminonucleoside; rhVEGF, recombinant human VEGF165; rtTA, reverse tetracycline transactivator; sFlt1, soluble fms-like tyrosine kinase 1; Tg, double-transgenic for podocin-rtTA and TRE-hVEGF; TRE, tetracycline responsive element; WT1, Wilms' tumor 1 
yielding glomerular purity $>97 \%$ used in primary podocyte culture or ELISA Podocytes (P0) outgrowing isolated glomeruli were identified by immunostainings for Wilms' tumor 1 (WT1; Santa Cruz Biotechnology, Santa Cruz, CA), nephrin (Progen, Germany) and synaptopodin (Progen). Only passage 3 (P3) podocytes were used in the in vitro studies.

To study effects of supplementing podocytes with recombinant human VEGF165 (rhVEGF; R\&D Systems, Minneapolis, MN), P3 podocytes were treated with low-serum $(2.5 \%)$ podocyte-conditioned medium and then with normal podocyte-conditioned medium containing rhVEGF $(10 \mathrm{ng} / \mathrm{mL})$, PAN (100 $\mu \mathrm{g} / \mathrm{mL}$; Sigma Chemical Co.-Aldrich, St. Louis, MO), rhVEGF plus PAN, or PBS control for $48 \mathrm{~h}$.

As our preliminary experiments showed slightly increased expression of cytoskeletal proteins in cultured podocytes with Dox treatment, to study the protective role of podocyte-derived VEGF against PAN-induced injury, paired Tg and Ctrl podocytes were both treated with Dox $(1 \mu \mathrm{g} / \mathrm{mL}$; Sigma Chemical Co.-Aldrich) with or without PAN for $48 \mathrm{~h}$.

PAN-induced podocyte injury in maturing mice. To induce hVEGF transgene expression, podocin-rtTA and TRE-hVEGF neonatal mice were treated with Dox $(2 \mathrm{mg} / \mathrm{mL}$ in drinking water containing $1 \%$ sucrose $)$ through the nursing dam starting on the day of delivery. PAN $(50 \mu \mathrm{g} / \mathrm{g}$ body weight, IP) or normal saline was injected $1 \mathrm{~d}$ after birth. Blood and urine were collected and kidneys harvested at $3 \mathrm{wk}$.

ELISA, Western blot analysis, and quantitative RT-PCR. Albuminuria was determined by urinary albumin to creatinine ratio (Exocell, Philadelphia, PA). Human or mouse-specific VEGF and soluble VEGF receptor 1, also known as soluble fms-like tyrosine kinase 1 (sFlt1), were measured by ELISA $(R \& D)$. Protein expression was assessed by Western blotting normalized to $\beta$-actin and synaptopodin mRNA by quantitative RT-PCR normalized to $18 \mathrm{~s}$ rRNA (26).

Immunocytochemistry and immunohistochemistry. Cells grown on slides were fixed in acetone, incubated with anti-synaptopodin, anti-WT1 (Santa Cruz), or anti-nephrin, followed by appropriate fluorescent secondary antibody. For F-actin staining of cultured podocytes, Alexa Fluor 546-labeled phalloidin (Invitrogen) was used. For immunohistochemistry, standard ABC method (Vector Labs, Burlingame, CA) was applied on paraffin-embedded kidney sections using anti-desmin (Abcam, UK), anti-nephrin, anti-WT1, anti-human VEGF (R\&D), anti-human/mouse VEGF (Zymed, San Francisco, CA), or anti-mouse VEGF (Sigma Chemical Co.).

Glomerular morphometry. On each paraffin-embedded kidney section stained with WT1, $>30$ glomeruli were analyzed by ImageJ software (National Institutes of Health) and assessed for glomerular volume $\left(V_{\text {glom }}\right)$, and

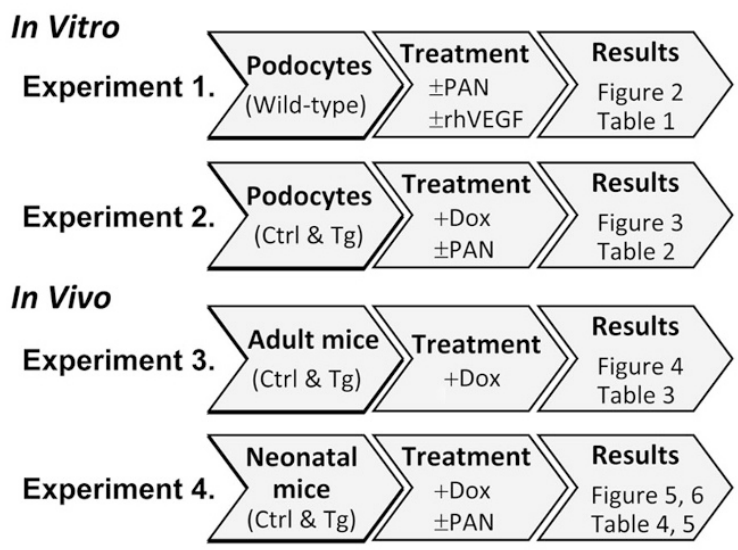

Figure 1. Experimental design. density and number of WT1-positive podocytes by stereologic morphometrics (27-29). Glomerular epithelial injury shown by desmin staining was graded on a $0-4$ scale, which represents the positive area involving $0 \%, 1-25 \%$, $26-50 \%, 51-75 \%$, or $>75 \%$ of the glomerular epithelium. For nephrinstained sections, all superficial glomeruli were analyzed by AxioVision software and percentage of positive area in the glomerulus averaged for each mouse. Measurements were validated separately by laboratory staff without knowledge of the groups.

Statistical analysis. Data were expressed as mean \pm SEM. Mann-Whitney $U$ tests were used for between-group comparisons and Wilcoxon signed-rank tests for paired comparisons. All statistical tests were two-sided, and significance was defined as $p<0.05$. Statistical analysis was performed using SPSS.

\section{RESULTS}

Effects of exogenous VEGF and PAN on podocyte VEGF (in vitro). Primary cultured mouse podocytes from WT adult mice were exposed to PAN or vehicle, and/or rhVEGF for $48 \mathrm{~h}$. The supernatant concentration of hVEGF was $1 \mathrm{ng} / \mathrm{mL}$, a level that was $3.79 \pm 0.12$ fold of mouse VEGF-A (mVEGF). Furthermore, mVEGF levels in the supernatant or cell lysates were unaffected by supplementation with rhVEGF (Table 1), suggesting that rhVEGF supplementation does not affect endogenous mVEGF. PAN significantly decreased podocyte $\mathrm{mVEGF}$ and significantly reduced $\mathrm{hVEGF}$ in lysates of cells treated with rhVEGF (Table 1). These results indicate that injured podocytes have reduced capacity to produce en-

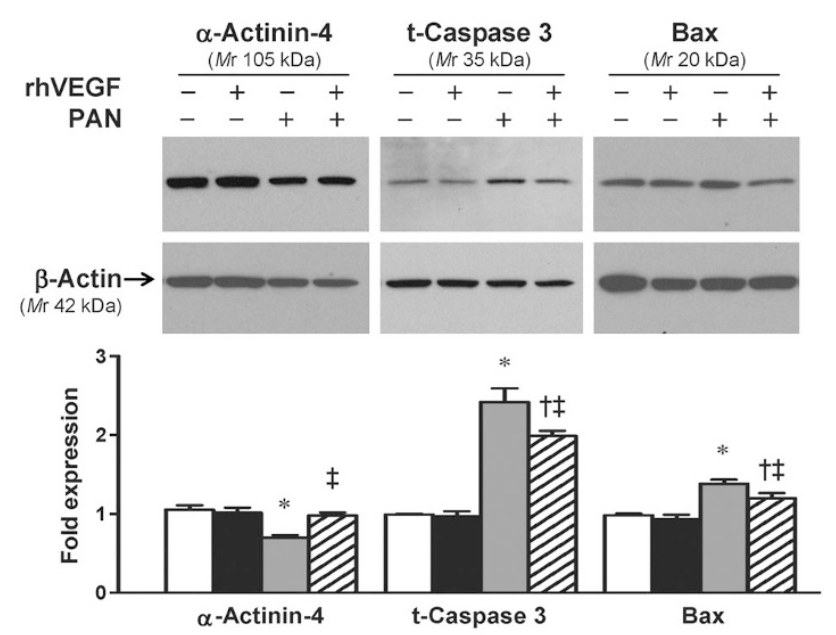

Figure 2. Effects of rhVEGF on PAN-exposed cultured podocytes. PAN significantly decreased expression of podocyte slit diaphragm protein $\alpha$-actinin-4 and increased apoptotic markers total caspase 3 and Bax. VEGF supplementation restored suppressed $\alpha$-actinin- 4 and attenuated increased total caspase 3 (t-Caspase 3) and Bax. $\square$, control; $\mathbf{\square}$, rhVEGF; $\square$, PAN; $\mathbf{Z \text { , }}$ PAN + rhVEGF. ${ }^{*} p<0.05 v s$. Ctrl; $\dagger p<0.05 v s$. rhVEGF; $\ddagger p<0.05 v s$. PAN. Experiments used primary podocytes from $n=4$ mice.

Table 1. Cellular and supernatant VEGF concentrations in cultured podocytes exposed to PAN and/or rhVEGF

\begin{tabular}{|c|c|c|c|c|c|}
\hline & \multirow[b]{2}{*}{$n$} & \multicolumn{2}{|c|}{ Cell lysate } & \multicolumn{2}{|c|}{ Supernatant } \\
\hline & & hVEGF (pg/mg) & mVEGF (pg/mg) & hVEGF (pg/mg) & mVEGF (pg/mg) \\
\hline Control & 4 & $0 \pm 0$ & $431.4 \pm 6.1$ & $0 \pm 0$ & $272.7 \pm 20.1$ \\
\hline rhVEGF & 4 & $1493.6 \pm 198.2$ & $433.8 \pm 37.7$ & $1005.4 \pm 18.3$ & $278.5 \pm 9.9$ \\
\hline PAN & 4 & $0 \pm 0$ & $356.6 \pm 19.8^{*}$ & $0 \pm 0$ & $286.9 \pm 20.5$ \\
\hline PAN + rhVEGF & 4 & $477.4 \pm 160.6 \dagger$ & $310.3 \pm 57.2$ & $1073.8 \pm 29.4$ & $274.2 \pm 15.9$ \\
\hline
\end{tabular}

Data are presented as mean \pm SEM. rhVEGF, $10 \mathrm{ng} / \mathrm{mL}$; PAN, $100 \mu \mathrm{g} / \mathrm{mL}$.

$* p<0.05$ vs. Ctrl.

$\dagger p<0.05$ vs. rhVEGF. 
dogenous VEGF and have an impaired ability to interact with and/or take up supplemental VEGF.

PAN significantly reduced expression of the podocyte cytoskeleton protein $\alpha$-actinin- 4 and increased expression of the apoptosis marker total caspase 3 and Bax. Supplementation of rhVEGF significantly attenuated these injury-related changes (Fig. 2).

Effects of podocyte-derived VEGF on PAN-injured podocytes (in vitro). To study whether enhanced intrinsic podocyte-derived VEGF protects against injury, mouse podocytes double-transgenic for podocin-rtTA and TRE-hVEGF were exposed to PAN. Induction of hVEGF-A transgene was achieved by Dox. Induced hVEGF was detected in the supernatant of $\mathrm{Tg}$ podocytes as early as $1 \mathrm{~h}$ after Dox and was sustained. By contrast, hVEGF was not detected in Doxtreated WT or podocin-rtTA single transgenic podocytes (Ctrl podocytes). Intrinsic mVEGF expression and secretion were not significantly changed by induced expression of podocytederived hVEGF in Tg podocytes compared with Ctrl podo-

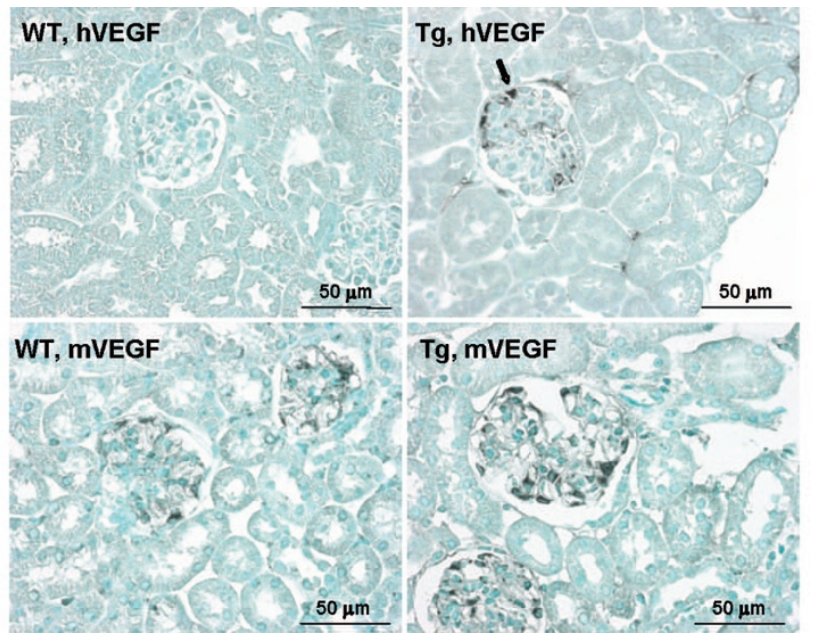

Figure 4. Immunostaining for hVEGF and mVEGF on the kidney specimens from Dox-treated Tg mice. Expression of hVEGF transgene was detected in Tg glomeruli but not in WT. Expression of intrinsic mVEGF in podocytes was not affected by hVEGF transgene expression. Arrow indicates a podocyte with positive hVEGF staining.

Table 2. Cellular and supernatant VEGF concentrations in cultured Ctrl and Tg podocytes exposed to PAN

\begin{tabular}{|c|c|c|c|c|c|}
\hline & \multirow[b]{2}{*}{$n$} & \multicolumn{2}{|c|}{ Cell lysate } & \multicolumn{2}{|c|}{ Supernatant } \\
\hline & & hVEGF (pg/mg) & mVEGF (pg/mg) & hVEGF (pg/mg) & mVEGF $(\mathrm{pg} / \mathrm{mg})$ \\
\hline Ctrl & 4 & $0 \pm 0$ & $412.2 \pm 22.5$ & $0 \pm 0$ & $240.2 \pm 9.7$ \\
\hline $\mathrm{Tg}$ & 4 & $2929.0 \pm 610.6$ & $448.9 \pm 3.9$ & $1425.9 \pm 99.8$ & $283.5 \pm 9.4$ \\
\hline $\mathrm{Ctrl}+\mathrm{PAN}$ & 4 & $0 \pm 0$ & $246.8 \pm 0.7^{*}$ & $0 \pm 0$ & $284.4 \pm 19.6$ \\
\hline $\mathrm{Tg}+\mathrm{PAN}$ & 4 & $126.9 \pm 54.1 \dagger$ & $289.6 \pm 5.2 \dagger \dagger$ & $736.5 \pm 199.5 \dagger$ & $269.8 \pm 8.0$ \\
\hline
\end{tabular}

Data are presented as mean \pm SEM. All cells were treated with Dox.

$* p<0.05$ vs. Ctrl.

$\dagger p<0.05$ vs. Tg.

$\ddagger p<0.05$ vs. Ctrl + PAN.
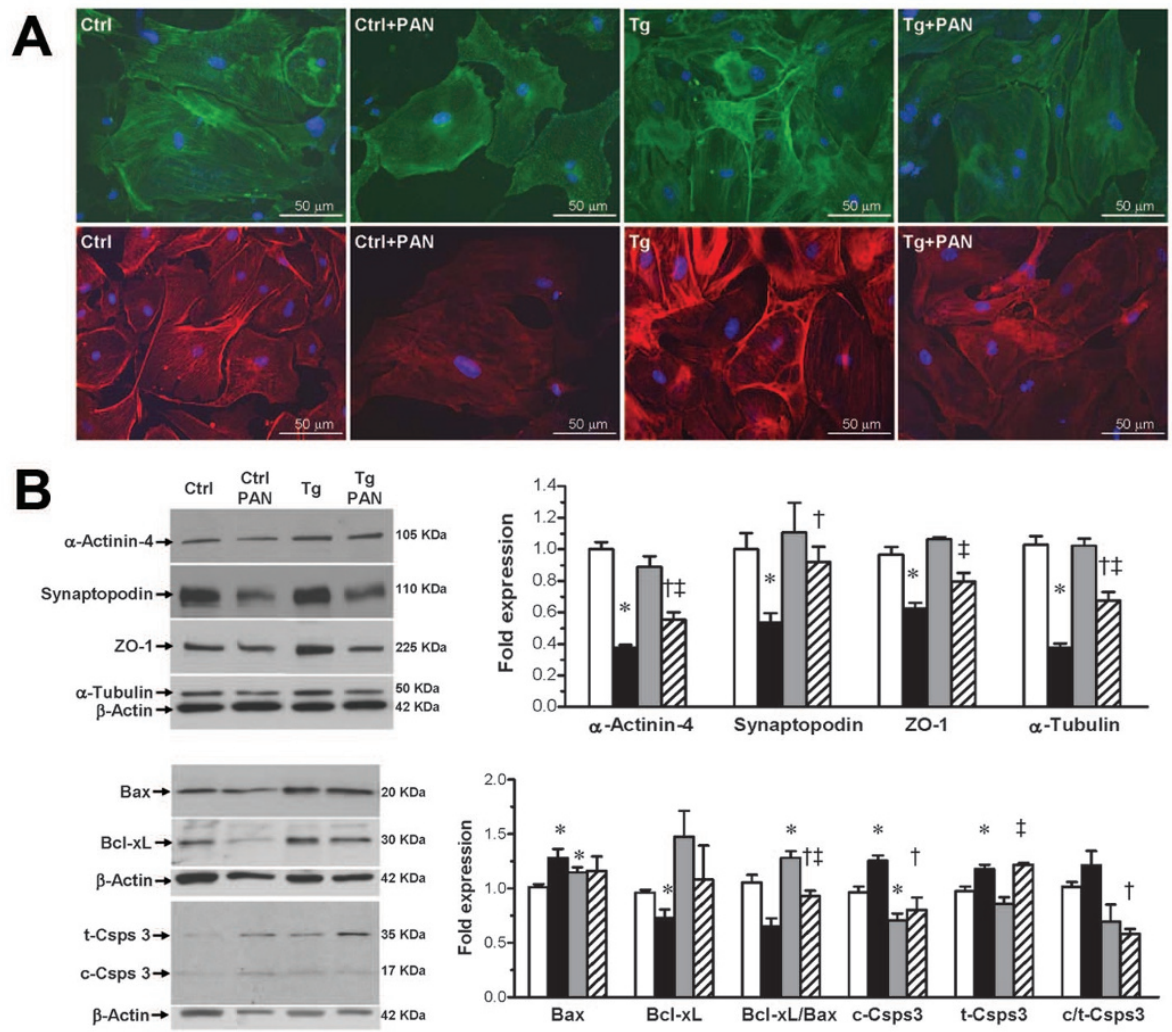

Figure 3. Effects of induced expression of podocyte-derived VEGF on PAN-injured podocytes. (A) PAN caused retraction of podocyte processes, and it significantly down-regulated and disrupted synaptopodin (green) and F-actin (red) in both $\mathrm{Ctrl}$ and $\mathrm{Tg}$ podocytes. Induction of podocyte-derived VEGF attenuated PANinduced suppression of synaptopodin and F-actin in $\mathrm{Tg}$ podocytes as well as lessening process retraction. (B) Enhancement of podocyte VEGF expression in $\mathrm{Tg}$ podocytes significantly ameliorated suppression of $\alpha$-actinin- 4 , synaptopodin, and $\alpha$-tubulin caused by PAN when compared with Ctrl. In Ctrl podocytes, PAN significantly increased expression of apoptotic marker cleaved caspase 3 (c-Csps 3), total caspase 3 (t-Csps 3), and Bax and decreased antiapoptotic $\mathrm{Bcl}-\mathrm{xL}$ and ratio of $\mathrm{Bcl}-\mathrm{xL} / \mathrm{Bax}$. Induction of podocyte-derived VEGF expression significantly attenuated PAN-induced changes in the ratio of c-Csps 3 to $\mathrm{t}-\mathrm{Csps} 3$ (c/t-Csps 3) and Bcl-xL/Bax. $\square$, Ctrl; $\boldsymbol{\square}, \mathrm{Ctrl}+\mathrm{PAN} ; \square, \mathrm{Tg} ; \boldsymbol{Z}$, Tg + PAN. * $p<0.05$ vs. Ctrl; $\nmid p<0.05$ vs. Tg; $\ddagger p<0.05$ vs. Ctrl + PAN. All podocytes were treated with Dox. Podocytes from $n=4$ pairs of Ctrl and Tg mice. 
cytes. The total human and mouse VEGF levels were some 6.5-fold higher in $\mathrm{Tg}$ podocyte lysate and 5.0-fold in $\mathrm{Tg}$ supernatant than the Ctrl.

PAN significantly down-regulated expression of mVEGF in Tg and Ctrl podocytes and hVEGF in Dox-treated Tg podocytes. However, Dox-treated $\mathrm{Tg}$ podocytes with induced podocyte-derived VEGF significantly ameliorated reduction in mVEGF (Table 2). Moreover, even $48 \mathrm{~h}$ after Dox, mVEGF levels in cell lysates and supernatants and hVEGF levels in supernatants were comparable between WT podocytes supplemented with rhVEGF and Dox-treated Tg podocytes with induced hVEGF (Tables 1 and 2).

PAN-exposed cultured podocytes showed retracted processes, disrupted and reduced cytoskeleton protein F-actin

Table 3. VEGF level and ratio of human/mouse VEGF in Tg mouse glomeruli and kidneys treated with Dox

\begin{tabular}{lcccc}
\hline & & hVEGF & mVEGF & human/mouse \\
& $n$ & (pg/mg protein) & (pg/mg protein) & VEGF \\
\hline Glomerulus & 3 & $4834.2 \pm 1097.5$ & $1153.6 \pm 278.8$ & $4.21 \pm 0.27$ \\
Kidney & 3 & $39.2 \pm 1.2$ & $51.4 \pm 0.6$ & $0.60 \pm 0.16$ \\
\hline
\end{tabular}

Data are presented as mean \pm SEM.

Table 4. VEGF level in Ctrl and Tg neonatal kidneys treated with Dox and/or PAN

\begin{tabular}{lcccc}
\hline & & $\begin{array}{c}\text { hVEGF } \\
\text { (pg/mg protein })\end{array}$ & $\begin{array}{c}\text { mVEGF } \\
(\mathrm{pg} / \mathrm{mg} \text { protein })\end{array}$ & $\begin{array}{c}\text { Human/mouse } \\
\text { VEGF }\end{array}$ \\
\hline $\mathrm{Ctrl}$ & 3 & $0 \pm 0$ & $93.9 \pm 0.6$ & $0 \pm 0$ \\
$\mathrm{Tg}$ & 3 & $35.6 \pm 4.9$ & $92.8 \pm 4.8$ & $0.38 \pm 0.03$ \\
$\mathrm{Ctrl}+\mathrm{PAN}$ & 3 & $0 \pm 0$ & $107.6 \pm 12.5$ & $0 \pm 0$ \\
$\mathrm{Tg}+\mathrm{PAN}$ & 3 & $10.0 \pm 1.6^{*}$ & $128.1 \pm 12.0$ & $0.077 \pm 0.006^{*}$ \\
\hline
\end{tabular}

Data are presented as mean \pm SEM.

$* p<0.05$ vs. Tg.
(Fig. 3A), as well as down-regulated slit diaphragm protein synaptopodin, tight junction protein $\mathrm{ZO}-1$, and cytoskeleton protein $\alpha$-actinin- 4 and $\alpha$-tubulin. Tg podocytes with elevated podocyte VEGF had significantly ameliorated all these changes (Fig. 3B). PAN also significantly increased apoptosis marker cleaved and total caspase 3 , and tended to increase proapoptotic Bax and decrease antiapoptotic Bcl-xL in Ctrl podocytes. Tg podocytes with elevated podocyte VEGF had significantly preserved ratios of Bcl-xL/Bax and cleaved/totalcaspase 3 (Fig. 3B).

Western blot analysis showed significantly up-regulated expression of VEGF receptor 1, also known as fms-like tyrosine kinase 1 (Flt1), in Tg podocytes with elevated podocyte VEGF but down-regulated in PAN-exposed podocytes. Induction of hVEGF expression significantly attenuated the change in Flt1 expression caused by PAN (Ctrl, $1.00 \pm 0.04$; Tg, $2.08 \pm$ 0.07; Ctrl + PAN, $0.59 \pm 0.08 ; \mathrm{Tg}+$ PAN, $0.94 \pm 0.11$ fold change; all $p<0.05$ for Tg versus Ctrl, Ctrl + PAN versus $\mathrm{Ctrl}$, and $\mathrm{Tg}+\mathrm{PAN}$ versus $\mathrm{Ctrl}+\mathrm{PAN})$.

VEGF in podocin-rtTA/TRE-hVEGF double-transgenic mouse kidneys (in vivo). To determine the transgene expression in vivo, immunostaining and ELISA for humanspecific VEGF were assessed. Human VEGF staining on kidney sections from adult mice treated with Dox for 1-4 wk showed positive transgene expression primarily in glomerular podocytes. Increased expression of podocyte $\mathrm{hVEGF}$ in $\mathrm{Tg}$ mice did not affect the level of intrinsic mVEGF (Fig. 4), with an average of $1076.6 \pm 257.3 \mathrm{pg} / \mathrm{mg}$ protein in Ctrl glomeruli versus $1153.6 \pm 278.8 \mathrm{pg} / \mathrm{mg}$ protein in Tg glomeruli measured by ELISA (each $n=3$, $p>0.05$ ). Quantitation of VEGF expression in isolated glomeruli showed that the amount of hVEGF was some 4-fold of mVEGF (Table 3).

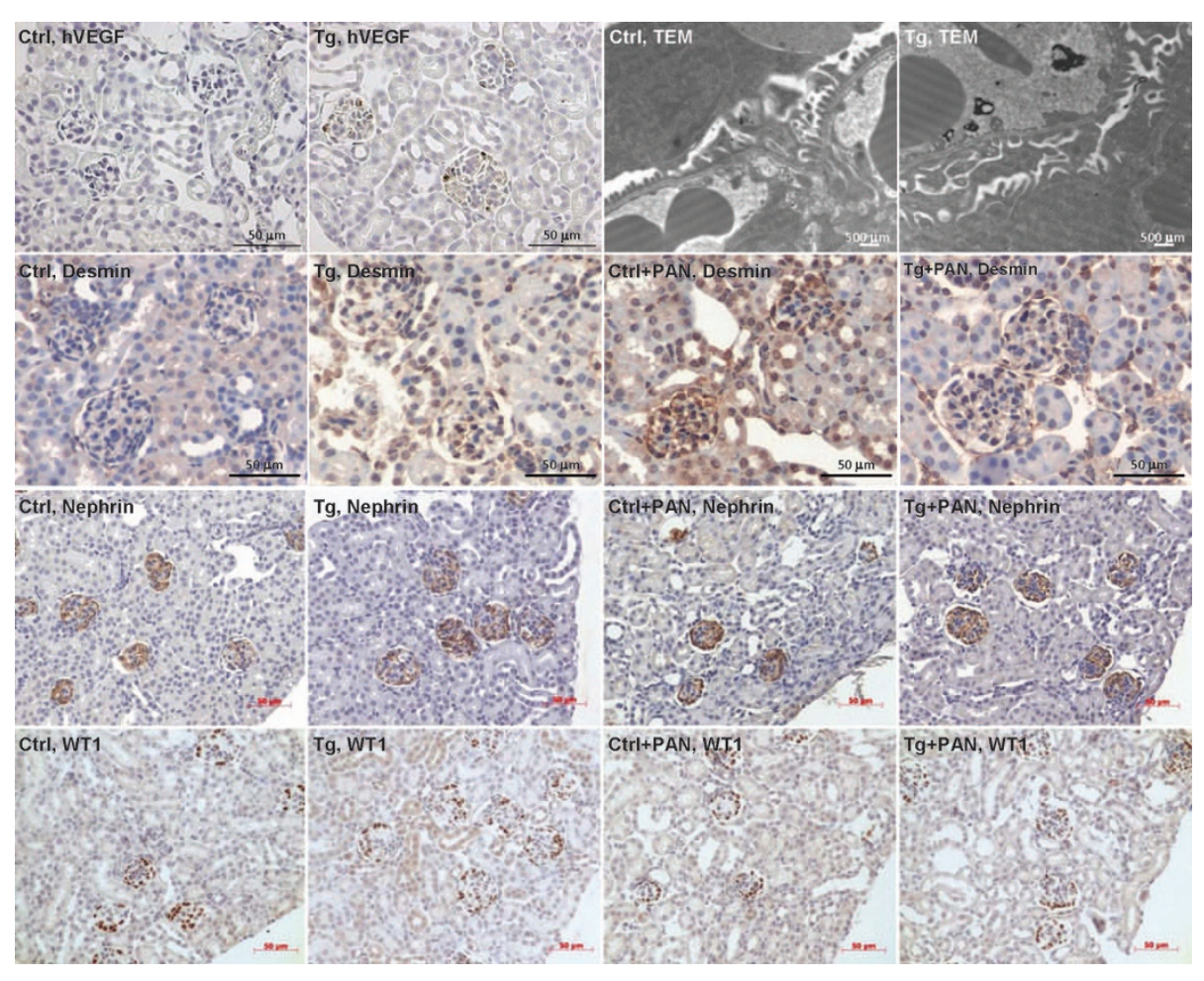

Figure 5. Renal morphology of neonatal Ctrl or Tg mice with or without PAN. hVEGF expression was only detected in $\mathrm{Tg}$ mice but not in the Ctrl. Tg mice with induced podocyte VEGF showed larger glomeruli than Ctrl and similar podocyte foot processes, glomerular basement membrane, and endothelial fenestrae examined by transmission electron microscopy (TEM). PAN-injected mice showed increased desmin and decreased nephrin expression, as well as smaller glomeruli with simpler branches of capillaries and increased density of WT1-positive podocytes in superficial glomeruli. These changes were attenuated by induction of podocyte-derived VEGF. 
Effects of induction in podocyte VEGF on PAN podocyte injury (in vivo). hVEGF expression was observed in kidneys of 3-wk-old Dox-treated mice (Table 4), localizing in podocytes (Fig. 5) of Tg but not Ctrl pups. Compared with the Ctrl, Dox-treated Tg mice with induced podocyte VEGF had larger glomeruli (Figs. 5 and 6A), greater nephrin-positive glomerular areas (Table 5; Fig. 5), and increased synaptopodin mRNA (Fig. 6B). Histological assessment revealed no structural damage in the glomerulus by induced podocyte VEGF
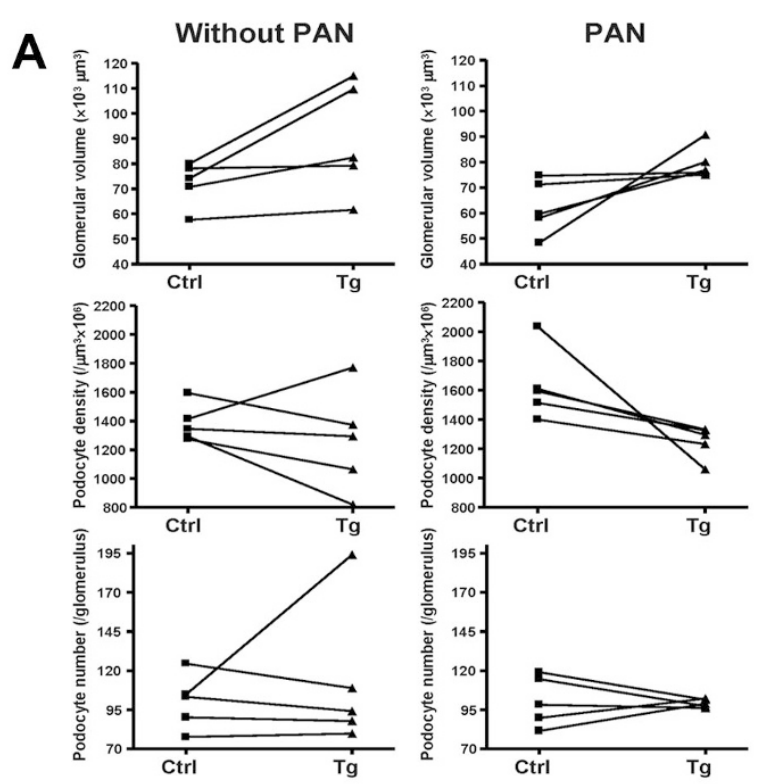

B

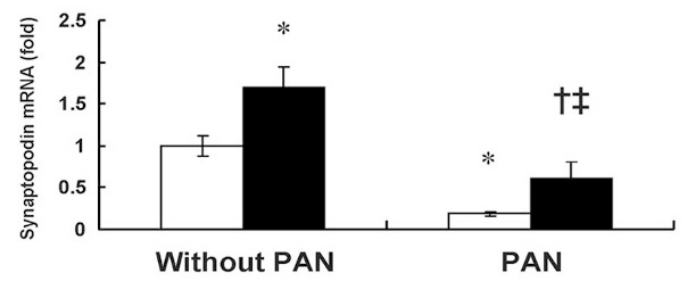

Figure 6. Changes in glomerular size and podocytes. (A) Ctrl and $\mathrm{Tg}$ littermates with or without PAN were paired according to the body weight and gender. Tg mice with induced podocyte-derived VEGF had significantly larger glomeruli than their paired Ctrl in both PAN and vehicle groups (both $p<0.05$ ). Glomerular podocyte density that encompasses the podocyte number over glomerular volume was significantly reduced by enhanced podocyte VEGF in PAN-exposed mice $(p<0.05)$. Tg and Ctrl pairs for PAN treated or untreated $(n=5)$. (B) Enhanced podocyte-derived VEGF significantly increased synaptopodin expression in kidneys of $\mathrm{Ctrl}$ and significantly attenuated synaptopodin decrease caused by PAN injury. $\square, \mathrm{Ctrl} ; \boldsymbol{\square}, \mathrm{Tg} .{ }^{*} p<0.05$ vs. Ctrl; $\dagger p<0.05 v s$. Tg; $\ddagger p<0.05 v s$. Ctrl + PAN. Ctrl $(n=6)$ and $\mathrm{Tg}(n=7)$ without PAN, and Ctrl $(n=7)$ and $\operatorname{Tg}(n=7)$ with PAN.
(8). These findings were supported by transmission electron microscopy, which also showed no appreciable differences in podocyte foot processes, glomerular basement membrane, and endothelial fenestrae between $\mathrm{Ctrl}$ and $\mathrm{Tg}$ mice (Fig. 5).

PAN significantly reduced hVEGF expression in kidney homogenate of Tg pups (Table 4). PAN also lessened renal synaptopodin mRNA expression in both $\mathrm{Ctrl}$ and $\mathrm{Tg}$ mice. These changes were significantly alleviated by induction of podocyte VEGF in Tg mice (Fig. 6). Glomerular increase in desmin and decrease in nephrin induced by PAN were attenuated by enhanced expression of podocyte VEGF (Table 5; Fig. 5). Glomeruli of PAN-exposed Ctrl mice tended to have smaller glomerular volume (Table $5 ; p=0.094$ ) with simpler branching of capillaries and higher podocyte density (Table 5; $p=0.064)$ than non-PAN-treated Ctrl. Pairing Tg and Ctrl mice from the same litter according to the body weight revealed that induced expression of podocyte-derived VEGF increased glomerular volume in both PAN-exposed and nonPAN-exposed mice and decreased podocyte density in PANexposed mice (Fig. 6A). At 3 wk, ELISA analysis of urine for albumin showed no proteinuria in $\mathrm{Tg}$ and $\mathrm{Ctrl}$ mice treated with Dox with or without PAN (Ctrl, $81.1 \pm 31.3$; Tg, $38.4 \pm$ 12.3; Ctrl + PAN, $65.9 \pm 22.1 ; \mathrm{Tg}+$ PAN, $35.1 \pm 11.4$ $\mu \mathrm{g} / \mathrm{mg}$ creatinine) $(8,21,30)$. Plasma creatinine measured by HPLC was not significantly different among the groups (Ctrl, $0.105 \pm 0.024 ; \mathrm{Tg}, 0.102 \pm 0.026 ; \mathrm{Ctrl}+\mathrm{PAN}, 0.116 \pm$ $0.011 ; \mathrm{Tg}+\mathrm{PAN}, 0.114 \pm 0.018 \mathrm{mg} / \mathrm{dL}$ ). As expected during this rapid maturational growth stage, apoptotic signals and apoptotic cells were rarely detected by TUNEL or caspase staining in the 3-wk mouse kidneys with or without PAN exposure.

\section{DISCUSSION}

Using in vitro and in vivo transgenic models of inducible podocyte-specific VEGF, we demonstrate a protective effect of intrinsic VEGF against podocyte injury and suggest that podocyte-derived VEGF has a self-preserving autocrine function. Our data also indicate that augmentation of intrinsic podocyte VEGF within the physiological range can abort deterioration of glomerular development prompted by podocyte injury.

In our study, the podocyte-derived VEGF was increased by a maximum 6-fold of the intrinsic mVEGF in cultured podocytes (Table 2) and 4-fold in isolated glomeruli from Tg mice (Table 3). This level of increase parallels that observed in studies with hypoxia which induced 3- to 7-fold increase in VEGF-A expression or secretion in cultured cells (31-34).

Table 5. Glomerular histology in podocin-rtTA/TRE-hVEGF double-transgenic kidneys treated with Dox and/or PAN

\begin{tabular}{llllcrl}
\hline & $n$ & Desmin score & Nephrin $(\%)$ & $N_{\mathrm{V}}\left(/ \mu \mathrm{m}^{3} \times 10^{6}\right)$ & Podocytes $(/$ glomerulus $)$ & Glomerular volume $\left(\mu \mathrm{m}^{3}\right)$ \\
\hline Ctrl & 7 & $0.51 \pm 0.14$ & $3.99 \pm 0.41$ & $1,424 \pm 59$ & $101.2 \pm 5.5$ & $71,347 \pm 3,528$ \\
Tg & 9 & $0.79 \pm 0.13$ & $5.20 \pm 0.25^{*}$ & $1,512 \pm 138$ & $119.6 \pm 12.8$ & $80,740 \pm 6,564$ \\
Ctrl + PAN & 7 & $1.64 \pm 0.16^{*}$ & $2.98 \pm 0.15^{*}$ & $1,622 \pm 81$ & $100.3 \pm 5.4$ & $62,345 \pm 3,466$ \\
Tg + PAN & 7 & $0.79 \pm 0.24 \dagger$ & $4.06 \pm 0.41 \dagger \neq$ & $1,358 \pm 78 \dagger$ & $92.2 \pm 3.7$ & $70,011 \pm 6,077$ \\
\hline
\end{tabular}

Data are presented as mean \pm SEM. $N_{\mathrm{V}}$ denotes glomerular podocyte density.

$* p<0.05$ vs. Ctrl.

$\dagger p<0.05$ vs. Ctrl + PAN.

$\$ p<0.05$ vs. Tg. 
In vivo, VEGF was found 5- to 10-fold higher after hypoxia in the retina (35), in the cornea with neovascularization (36), and in tumor tissues (37). Thus, data of our study indicated that the magnitude of increase in podocyte VEGF in the Tg mice was in the physiological rather than pharmacological range. Notably, this physiological level of VEGF induction did not result in proteinuria, glomerular endothelial injury, or glomerulosclerosis $(8,30)$. Moreover, both ELISA measurement of cultured podocytes and isolated glomeruli indicated that the supplemental hVEGF transgene expression does not affect the level of intrinsic mVEGF.

PAN has been shown to cause derangement and downregulation of cytoskeletal proteins as well as increased apoptosis in cultured podocytes $(20,38)$, and podocyte foot process effacement and altered expression/localization of slit diaphragm proteins in vivo $(38,39)$. In our study on primary podocytes, these changes were found associated with a significant decrease in cellular VEGF after PAN injury. The level of recombinant VEGF was also greatly reduced compared with podocytes without PAN injury. Therefore, PAN injury compromises podocyte survival by down-regulating intrinsic VEGF expression and/or reducing the cell's capability to use VEGF from adjacent podocytes. It is not known whether the turnover of VEGF is changed in diseased podocytes and whether higher dose of rhVEGF exerts greater protective effects. Given that the nature of podocyte deterioration follows an autonomous vicious cycle, our data suggest that supplementation of intrinsic VEGF protects podocytes by disrupting this injurious cycle (19).

Previous studies in glomerular injury models $(40,41)$ suggested an antagonistic effect of soluble VEGF receptor 1 (sFlt1) on podocyte preservation, while decreased Flt1 expression has been linked to increased apoptosis $(42,43)$. The sFlt1 levels in cultured podocyte supernatant in the current study were not significantly different after PAN and/or induction of VEGF expression. However, the Flt1 expression was decreased by PAN and increased in podocytes with induced hVEGF with or without PAN. Thus, the preserved Flt1 expression in PAN-injured podocytes may mediate the autocrine protective effects of VEGF against podocyte injury. Although VEGF receptor 2 (VEGFR-2) is regarded as functional receptor of VEGF, our study did not find convincing changes in its phosphorylation or expression in cultured podocytes (data not shown), which is consistent with a most recent finding that autocrine effects of podocyte VEGF may not actively involve VEGFR-2 (44).

In vivo, mouse podocytes are sensitive to PAN only during the developmental stage (21). Our previous study showed transient effacement of podocyte foot processes without proteinuria in neonatal mice after PAN injection (21). The injured podocytes cause abnormal development of glomerular capillaries with simpler branching and reduced glomerular endothelial volume, changes that are more prominent in superficial than deep glomeruli (21). In developing mice, we detected a significantly reduced expression of podocyte-specific synaptopodin and nephrin as well as an increase in desmin after PAN injury while no change in mVEGF likely due to contribution from its nonpodocyte sources. Enhanced expression of podocyte-derived VEGF not only significantly preserved synaptopodin and nephrin expression but also dampened upregulated desmin after PAN. These data indicate a selfprotective role of podocyte VEGF against injury and propagation of injury in vivo. Previous studies have shown that glomerular development is influenced by podocytederived VEGF $(8,45)$. In the current study, enhanced podocyte-derived VEGF caused larger glomerular volume in normally developing uninjured mice, whereas supplementing podocyte VEGF preserved glomerular growth deteriorated by PAN injury. The effects of enhanced podocyte VEGF on glomerular development also suggest a proangiogenic form of hVEGF rather than antiangiogenic splice variants (46), and a paracrine effect of VEGF on endothelial cells in developing glomeruli (44). The nonsignificant changes in podocyte density or number between the normal mice with and without enhanced podocyte VEGF, and the restored podocyte density in PAN-injured mice with enhanced podocyte VEGF, together with increased synaptopodin and nephrin expression, indicate that the enhanced expression of podocyte VEGF may be conducive for podocyte adaptation that occurs in parallel with enlargement of the glomerular vasculature under pathophysiological conditions.

In summary, our studies confirm an autocrine survival effect of VEGF in podocytes in vitro (47-49) and demonstrate protective roles of enhanced intrinsic VEGF against podocyte injury and in preservation of normal glomerular development in vivo.

\section{REFERENCES}

1. Kriz W 2002 Podocyte is the major culprit accounting for the progression of chronic renal disease. Microsc Res Tech 57:189-195

2. D'Agati VD 2008 The spectrum of focal segmental glomerulosclerosis: new insights. Curr Opin Nephrol Hypertens 17:271-281

3. Pollak MR 2008 Focal segmental glomerulosclerosis: recent advances. Curr Opin Nephrol Hypertens 17:138-142

4. Byrne AM, Bouchier-Hayes DJ, Harmey JH 2005 Angiogenic and cell survival functions of vascular endothelial growth factor (VEGF). J Cell Mol Med 9:777-794

5. Spyridopoulos I, Brogi E, Kearney M, Sullivan AB, Cetrulo C, Isner JM, Losordo DW 1997 Vascular endothelial growth factor inhibits endothelial cell apoptosis induced by tumor necrosis factor-alpha: balance between growth and death signals. J Mol Cell Cardiol 29:1321-1330

6. Simon M, Grone HJ, Johren O, Kullmer J, Plate KH, Risau W, Fuchs E 1995 Expression of vascular endothelial growth factor and its receptors in human renal ontogenesis and in adult kidney. Am J Physiol 268:F240-F250

7. Eremina V, Jefferson JA, Kowalewska J, Hochster H, Haas M, Weisstuch J, Richardson C, Kopp JB, Kabir MG, Backx PH, Gerber HP, Ferrara N, Barisoni L, Alpers CE, Quaggin SE 2008 VEGF inhibition and renal thrombotic microangiopathy. N Engl J Med 358:1129-1136

8. Eremina V, Sood M, Haigh J, Nagy A, Lajoie G, Ferrara N, Gerber HP, Kikkawa Y, Miner JH, Quaggin SE 2003 Glomerular-specific alterations of VEGF-A expression lead to distinct congenital and acquired renal diseases. J Clin Invest 111:707-716

9. Kang DH, Hughes J, Mazzali M, Schreiner GF, Johnson RJ 2001 Impaired angiogenesis in the remnant kidney model: II. Vascular endothelial growth factor administration reduces renal fibrosis and stabilizes renal function. J Am Soc Nephrol 12:1448-1457

10. Kelly DJ, Hepper C, Wu LL, Cox AJ, Gilbert RE 2003 Vascular endothelial growth factor expression and glomerular endothelial cell loss in the remnant kidney model. Nephrol Dial Transplant 18:1286-1292

11. Masuda Y, Shimizu A, Mori T, Ishiwata T, Kitamura H, Ohashi R, Ishizaki M, Asano G, Sugisaki Y, Yamanaka N 2001 Vascular endothelial growth factor enhances glomerular capillary repair and accelerates resolution of experimentally induced glomerulonephritis. Am J Pathol 159:599-608

12. Boner G, Cox AJ, Kelly DJ, Tobar A, Bernheim J, Langham RG, Cooper ME, Gilbert RE 2003 Does vascular endothelial growth factor (VEGF) play a role in the pathogenesis of minimal change disease? Nephrol Dial Transplant 18:2293-2299

13. Honkanen E, von Willebrand E, Koskinen P, Teppo AM, Tornroth T, Ruutu M, Gronhagen-Riska C 2003 Decreased expression of vascular endothelial growth factor in idiopathic membranous glomerulonephritis: relationships to clinical course. Am J Kidney Dis 42:1139-1148 
14. Lindenmeyer MT, Kretzler M, Boucherot A, Berra S, Yasuda Y, Henger A, Eichinger F, Gaiser S, Schmid H, Rastaldi MP, Schrier RW, Schlondorff D, Cohen CD 2007 Interstitial vascular rarefaction and reduced VEGF-A expression in human diabetic nephropathy. J Am Soc Nephrol 18:1765-1776

15. Jefferson JA, Shankland SJ, Pichler RH 2008 Proteinuria in diabetic kidney disease: a mechanistic viewpoint. Kidney Int 74:22-36

16. Mathieson PW 2007 Minimal change nephropathy and focal segmental glomerulosclerosis. Semin Immunopathol 29:415-426

17. Ronco P, Debiec H 2007 Target antigens and nephritogenic antibodies in membranous nephropathy: of rats and men. Semin Immunopathol 29:445-458

18. Ziyadeh FN, Wolf G 2008 Pathogenesis of the podocytopathy and proteinuria in diabetic glomerulopathy. Curr Diabetes Rev 4:39-45

19. Ichikawa I, Ma J, Motojima M, Matsusaka T 2005 Podocyte damage damages podocytes: autonomous vicious cycle that drives local spread of glomerular sclerosis. Curr Opin Nephrol Hypertens 14:205-210

20. Wada T, Pippin JW, Marshall CB, Griffin SV, Shankland SJ 2005 Dexamethasone prevents podocyte apoptosis induced by puromycin aminonucleoside: role of p53 and Bcl-2-related family proteins. J Am Soc Nephrol 16:2615-2625

21. Ma J, Rossini M, Yang HC, Zuo Y, Fogo AB, Ichikawa I 2007 Effects of podocyte injury on glomerular development. Pediatr Res 62:417-421

22. Shigehara T, Zaragoza C, Kitiyakara C, Takahashi H, Lu H, Moeller M, Holzman LB, Kopp JB 2003 Inducible podocyte-specific gene expression in transgenic mice. J Am Soc Nephrol 14:1998-2003

23. Ohno-Matsui K, Hirose A, Yamamoto S, Saikia J, Okamoto N, Gehlbach P, Duh EJ, Hackett S, Chang M, Bok D, Zack DJ, Campochiaro PA 2002 Inducible expression of vascular endothelial growth factor in adult mice causes severe proliferative retinopathy and retinal detachment. Am J Pathol 160:711-719

24. Takemoto M, Asker N, Gerhardt H, Lundkvist A, Johansson BR, Saito Y, Betsholtz C 2002 A new method for large scale isolation of kidney glomeruli from mice. Am J Pathol 161:799-805

25. Katsuya K, Yaoita E, Yoshida Y, Yamamoto Y, Yamamoto T 2006 An improved method for primary culture of rat podocytes. Kidney Int 69:2101-2106

26. Roselli S, Heidet L, Sich M, Henger A, Kretzler M, Gubler MC, Antignac C 2004 Early glomerular filtration defect and severe renal disease in podocin-deficient mice. Mol Cell Biol 24:550-560

27. Adamczak M, Gross ML, Amann K, Ritz E 2004 Reversal of glomerular lesions involves coordinated restructuring of glomerular microvasculature. J Am Soc Nephrol 15:3063-3072

28. Basgen JM, Nicholas SB, Mauer M, Rozen S, Nyengaard JR 2006 Comparison of methods for counting cells in the mouse glomerulus. Nephron Exp Nephrol 103:e139-e148

29. White KE, Bilous RW 2004 Estimation of podocyte number: a comparison of methods. Kidney Int 66:663-667

30. Belteki G, Haigh J, Kabacs N, Haigh K, Sison K, Costantini F, Whitsett J, Quaggin SE, Nagy 2005 A conditional and inducible transgene expression in mice through the combinatorial use of Cre-mediated recombination and tetracycline induction. $\mathrm{Nu}$ cleic Acids Res 33:e51

31. Dai Y, Xu M, Wang Y, Pasha Z, Li T, Ashraf M 2007 HIF-1alpha induced-VEGF overexpression in bone marrow stem cells protects cardiomyocytes against ischemia. J Mol Cell Cardiol 42:1036-1044

32. Matsumoto K, Obara N, Ema M, Horie M, Naka A, Takahashi S, Imagawa S 2009 Antitumor effects of 2-oxoglutarate through inhibition of angiogenesis in a murine tumor model. Cancer Sci 100:1639-1647
33. Naldini A, Morena E, Pucci A, Pellegrini M, Baldari CT, Pelicci PG, Presta M, Ribatti D, Carraro F 2010 The adaptor protein p66Shc is a positive regulator in the angiogenic response induced by hypoxic T cells. J Leukoc Biol 87:365-369

34. Dann JM, Sykes PH, Mason DR, Evans JJ 2009 Regulation of vascular endothelia growth factor in endometrial tumour cells by resveratrol and EGCG. Gynecol Oncol $113: 374-378$

35. Dal Monte M, Ristori C, Cammalleri M, Bagnoli P 2009 Effects of somatostatin analogues on retinal angiogenesis in a mouse model of oxygen-induced retinopathy: involvement of the somatostatin receptor subtype 2. Invest Ophthalmol Vis Sci 50:3596-3606

36. Kwon YS, Hong HS, Kim JC, Shin JS, Son Y 2005 Inhibitory effect of rapamycin on corneal neovascularization in vitro and in vivo. Invest Ophthalmol Vis Sci 46:454-460

37. Hendriksen EM, Span PN, Schuuring J, Peters JP, Sweep FC, van der Kogel AJ, Bussink J 2009 Angiogenesis, hypoxia and VEGF expression during tumour growth in a human xenograft tumour model. Microvasc Res 77:96-103

38. Eto N, Wada T, Inagi R, Takano H, Shimizu A, Kato H, Kurihara H, Kawachi H, Shankland SJ, Fujita T, Nangaku M 2007 Podocyte protection by darbepoetin: preservation of the cytoskeleton and nephrin expression. Kidney Int 72:455-463

39. Lee YK, Kwon T, Kim DJ, Huh W, Kim YG, Oh HY, Kawachi H 2004 Ultrastructural study on nephrin expression in experimental puromycin aminonucleoside nephrosis. Nephrol Dial Transplant 19:2981-2986

40. Ku CH, White KE, Dei Cas A, Hayward A, Webster Z, Bilous R, Marshall S, Viberti G, Gnudi L 2008 Inducible overexpression of sFlt-1 in podocytes ameliorates glomerulopathy in diabetic mice. Diabetes 57:2824-2833

41. Sugimoto H, Hamano Y, Charytan D, Cosgrove D, Kieran M, Sudhakar A, Kalluri R 2003 Neutralization of circulating vascular endothelial growth factor (VEGF) by anti-VEGF antibodies and soluble VEGF receptor 1 (sFlt-1) induces proteinuria. J Biol Chem 278:12605-12608

42. Zhu J, Wang S, Bie P, Li X, Zhang Y, Xiong Y, Wang H, Ma Z, Li K, Dong J 2007 Apoptosis and regeneration of sinusoidal endothelial cells after extended cold preservation and transplantation of rat liver. Transplantation 84:1483-1491

43. Fujita K, Nakayama M, Nakai Y, Takayama H, Nishimura K, Ujike T, Nishimura K, Aozasa K, Okuyama A, Nonomura N 2009 Vascular endothelial growth factor receptor 1 expression in pelvic lymph nodes predicts the risk of cancer progression after radical prostatectomy. Cancer Sci 100:1047-1050

44. Sison K, Eremina V, Baelde H, Min W, Hirashima M, Fantus IG, Quaggin SE 2010 Glomerular structure and function require paracrine, not autocrine, VEGF-VEGFR-2 signaling. J Am Soc Nephrol 21:1691-1701

45. Ueda H, Miyazaki Y, Matsusaka T, Utsunomiya Y, Kawamura T, Hosoya T, Ichikawa I 2008 BMP in podocytes is essential for normal glomerular capillary formation. J Am Soc Nephrol 19:685-694

46. Qiu Y, Ferguson J, Oltean S, Neal CR, Kaura A, Bevan H, Wood E, Sage LM, Lanati S, Nowak DG, Salmon AH, Bates D, Harper SJ 2010 Overexpression of VEGF165b in podocytes reduces glomerular permeability. J Am Soc Nephrol 21:1498-1509

47. Guan F, Villegas G, Teichman J, Mundel P, Tufro A 2006 Autocrine VEGF-A system in podocytes regulates podocin and its interaction with CD2AP. Am J Physiol Renal Physiol 291:F422-F428

48. Foster RR, Hole R, Anderson K, Satchell SC, Coward RJ, Mathieson PW, Gillat DA, Saleem MA, Bates DO, Harper SJ 2003 Functional evidence that vascular endothelial growth factor may act as an autocrine factor on human podocytes. Am J Physiol Renal Physiol 284:F1263-F1273

49. Müller-Deile J, Worthmann K, Saleem MA, Tossidou I, Haller H, Schiffer M 2009 The balance of autocrine VEGF-A and VEGF-C determines podocyte survival. Am J Physiol Renal Physiol 297:F1656-F1667 